\title{
O Paradigma das Políticas Públicas Baseadas em Evidências na Gestão Pública BRASILEIRA: UMA ANÁLISE DAS PUBLICAÇÕES ACADÊMICAS ${ }^{1}$
}

Tatiana Lemos Sandim²

Daienne Amaral Machado ${ }^{3}$

\section{INTRODUÇÃO}

Tem crescido nos últimos anos, no Brasil, o debate sobre a necessidade de basear as políticas públicas em evidências. Textos de advocacy têm ganhado espaço na mídia ${ }^{4}$ e nas redes sociais, cursos específicos têm sido criados nas escolas de administração pública ${ }^{5}$ e o próprio projeto de pesquisa $O$ que informa as politicas públicas federais: o uso e não uso de evidências pela burocracia federal brasileira indicam o crescente interesse do assunto na agenda pública.

O tema também chegou aos formuladores e implementadores de políticas públicas. $\mathrm{Na}$ administração pública federal, podem ser identificadas iniciativas promotoras da abordagem de políticas públicas baseadas em evidências (PPBEs), seja por meio do fomento à produção de estudos específicos (própria ou encomendada junto à academia), ou aproximando produtores de evidências dos gestores públicos, potenciais consumidores dessas evidências.

Exemplos de estratégias pioneiras são encontrados no atual Ministério da Cidadania, que conta com a Secretaria de Avaliação e Gestáo da Informaçáo, herdada do Ministério do Desenvolvimento Social e Combate à Fome e criada simultaneamente ao próprio ministério, em 2003; e no Ministério da Saúde, que, em 2009, patrocinou a criação da Rede para Políticas Públicas Informadas por Evidências (Evidence-Informed Policy Network - EvipNet). Mais recentemente, em 2016, foi criado o Comitê de Monitoramento e Avaliação de Políticas Públicas, pelo então Ministério do Planejamento, Desenvolvimento e Gestão, ${ }^{6}$ e, em 2018, o Ministério da Educação criou, junto ao gabinete do ministro, a Assessoria Estratégica de Evidências. A saúde é a área em que a discussão sobre o uso de evidências surgiu (Davies e Nutley, 1999) e, ainda hoje, parece ser a área em que estratégias para o uso de evidências têm maior espaço, inclusive no contexto internacional (Oliver et al., 2014).

Neste texto, entendemos que PPBEs é uma abordagem que pode ajudar gestores públicos a tomarem decisóes bem-informadas sobre políticas, programas e projetos, colocando a melhor evidência disponível no centro do processo de formulação e implementação dessas iniciativas (Davies, 2004). O que se pretende é ampliar as alternativas disponíveis, promovendo a reflexão sobre as consequências das açôes públicas sobre a sociedade (Carneiro e Danton, 2012). O objetivo da abordagem das PPBEs

\footnotetext{
1. DOI: http://dx.doi.org/10.38116/bapi24art3

2. Gerente de pesquisas na Diretoria de Estudos e Políticas Sociais da Companhia de Planejamento do Distrito Federal (Codeplan).

3. Diretora de estudos e políticas sociais da Codeplan.

4. Alguns exemplos estão disponíveis em: <https://www.nexojornal.com.br/colunistas/2018/Precisamos-de-pol\%C3\%ADticas-p\%C3\%BAblicascom-evid\%C3\%AAncias1>; <https://politica.estadao.com.br/blogs/blog-do-mlg/uso-de-evidencia-no-governo-do-que-estamos-falando/>; e $<$ https://www1.folha.uol.com.br/opiniao/2018/12/por-mais-evidencias-na-educacao.shtml>.

5. Por exemplo, o curso virtual da Escola Nacional de Administração Pública (Enap), em parceria com o The Abdul Latif Jameel Poverty Action Lab (J-PAL); o curso presencial da Enap; e o curso de gestão de políticas e projetos baseadas em evidências do Instituto de Ensino e Pesquisa (Insper). 6. Dele também faziam parte a Casa Civil da Presidência da República e a então Controladoria-Geral da União (CGU).
} 
não é subordinar a decisão política ao conhecimento científico. Evidências científicas integram o grupo de informaçóes de "boa qualidade" e ocupam posição central nessa abordagem, mas figuram ao lado de outros tipos de conhecimentos que geram insumos para a tomada de decisão de gestores e formuladores de políticas públicas (Head, 2008). Esse conjunto de conhecimentos diz respeito a recursos e dinâmicas de naturezas diferentes como correlaçóes múltiplas de forças sociais de diferentes segmentos, oportunidades políticas, questóes culturais, fiscais, orçamentárias e normativas.

A produção de evidências científicas úteis às políticas públicas brasileiras não é novidade. Universidades e institutos de pesquisa nacionais desenvolvem uma série de estudos, pesquisas e avaliaçóes sobre problemas sociais e sobre a própria atuação governamental. O governo federal produz muitos dados e evidências sobre sua atuação, por meio do próprio Ipea e de outros institutos de pesquisas. ${ }^{7}$ Governos estaduais também o fazem por meio de organizaçóes congêneres. ${ }^{8} \mathrm{O}$ que pode ter ganhado um impulso nas últimas décadas - e o que pretendemos investigar - são iniciativas deliberadas de colocar em prática, de formas mais estruturadas e talvez permanentes, a abordagem de políticas baseadas em evidências e elementos-chave dessas iniciativas.

Entendemos que a análise da produção acadêmica pode ser útil para elucidar as convergências entre o debate governamental e a agenda de pesquisa e pode colaborar para lançando mão de um trocadilho -, produzir evidências sobre como essa abordagem tem sido assimilada pela academia e por ela trabalhada. A estratégia metodológica empregada foi a busca bibliográfica em dissertaçóes, teses e artigos acadêmicos publicados em periódicos nacionais e internacionais sobre o contexto brasileiro. Teses e dissertaçóes foram incluídas para tentar apreender o debate mais recente, ainda não disponível em publicações revisadas por pares. Elucidamos, entretanto, que a publicação em livros ainda não foi incorporada nessa análise. Dada sua relevância para a divulgação da produção acadêmica, essa ausência poderá impactar os resultados encontrados.

\section{METODOLOGIA}

Para a busca por artigos, foram utilizados os seguintes grupos de palavras-chave nos repositórios de buscas Scielo e Periódicos Capes. ${ }^{9}$ Para ambos foi aplicado filtro de data para os últimos vinte anos (2000 a 2020).

Scielo: (ab:(("políticas públicas" OR "política pública" OR "gestão pública") AND (evidência OR evidências)) AND Brasil). Coleção: Brasil. Busca em: 10 fev. 2020.

Periódicos Capes: [no assunto]_ [contém] _ ["politic* public*" OR "public polic*" OR "gestao publica"]_ [AND] [Qualquer] _ [contém] _ [(Brazil OR Brasil*) AND ("basead* evidenci*" OR "evidence based")]. Periódicos revisados por pares. Busca em: 18 fev. 2020.

Para a busca de teses e dissertaçóes, foi utilizada a Biblioteca Digital Brasileira de Teses e Dissertaçóes (BDTD), que reúne mais de 600 mil documentos publicados em 120 instituiçóes nos últimos quinze anos. A busca realizada foi a seguinte.

Todos os campos: ("basead* em evidência*" OR "informad* por evidência*") E ("política* pública*" OR "gestão pública"). Busca realizada em: 8 maio 2020.

7. Outros exemplos são o Instituto Brasileiro de Geografia e Estatística (IBGE), o Instituto Nacional de Estudos e Pesquisas Educacionais Anísio Teixeira (Inep) e a Fundação Oswaldo Cruz (Fiocruz).

8. Para citar alguns, a Companhia de Planejamento do Distrito Federal (Codeplan), o Instituto de Pesquisa e Estratégia Econômica do Ceará (Ipece), a Fundação Sistema Estadual de Análise de Dados (Seade), de São Paulo, e a Fundação João Pinheiro (FJP), de Minas Gerais.

9. A busca foi feita utilizando-se um login para acesso completo ao conteúdo do portal. 
Foram identificados 334 artigos e 52 dissertações (37) e teses (15), tratados conforme apresentado na tabela 1 .

TABELA 1

Resultados da busca nos portais Scielo e Periódicos Capes

\begin{tabular}{lcccccc}
\hline Repositório & $\begin{array}{c}\text { Resultado } \\
\text { das buscas }\end{array}$ & Repetidos & $\begin{array}{c}\text { Excluídos após } \\
\text { leitura dos resumos }\end{array}$ & $\begin{array}{c}\text { Leitura diagonal } \\
\text { do texto }\end{array}$ & $\begin{array}{c}\text { Excluídos } \\
\text { após diagonal }\end{array}$ & $\begin{array}{c}\text { Incluídos para } \\
\text { leitura completa }\end{array}$ \\
\hline Scielo & 215 & 4 & 190 & 21 & 17 & 4 \\
Capes & 119 & 7 & 96 & 16 & 14 & 2 \\
BDTD & 52 & 3 & 44 & 42 & 32 & 4 \\
Total & 386 & 14 & 330 & & & \\
\hline
\end{tabular}

Elaboração das autoras.

Foi feita uma leitura diagonal ${ }^{10}$ dos 42 remanescentes. Após a leitura, parte deles também foi excluída, utilizando-se os mesmos critérios. A maioria das exclusões (133) aconteceu porque os artigos utilizam o termo "evidência científica" de forma ampla e normalizada em textos acadêmicos, sem relação com PPBEs. Outros 101 documentos utilizavam o verbo "evidenciar" para destacar algo, com frases como "evidencia-se que...". ${ }^{11}$ Os outros 51 estudos náo focalizavam o Brasil como objeto de estudo e 22 estudos não usavam o termo "evidência" e nem faziam referência às PPBEs.

Parte dos estudos excluídos mencionava as PPBEs de forma transversal (21), geralmente, na introdução, como parte da justificativa de sua relevância, ou nas conclusões, criando um diálogo entre os resultados e o desenvolvimento desejável de PPBEs. Entre as teses e dissertaçóes, sete documentos argumentavam a favor da abordagem das PPBEs como alternativa para o desenvolvimento de políticas públicas, sobretudo na saúde. Nesses casos, prática baseada em evidências figurava como pressuposto de uma intervenção adequada ou almejada. Entre os estudos no campo da saúde, também é encontrado o debate sobre "medicina baseada em evidências" e o uso de evidências nas práticas clínicas. Esses estudos foram excluídos porque não discutem o paradigma das PPBEs de forma mais ampla. Por fim, um grupo de estudos não tratava de políticas públicas. Em dois desses, ${ }^{12}$ por exemplo, propunham-se procedimentos para a construção de sínteses de evidências. ${ }^{13}$

Ao final, seis artigos e quatro dissertaçóes foram lidos integralmente e analisados. Os artigos foram publicados entre 2009 e 2019, nas áreas de saúde (4), educação (1) e sociologia (1). Os resultados são apresentados na próxima seção. As dissertações foram defendidas entre 2016 e 2019 nas áreas de políticas públicas (1), educação (1), ecologia (1) e saúde coletiva (1). Os poucos resultados identificados são um indicativo de que o crescente interesse pela abordagem na administraçáo pública, possivelmente, ainda não se reflete na agenda de pesquisa acadêmica.

10. Processo de revisão de literatura chamado skimming of the text. Consiste em identificar, sem uma leitura compreensiva do documento, se 0 texto atende aos critérios de inclusão.

11. Na linguagem acadêmica, é comum o uso desse verbo para destacar argumentos, em conformidade com sua definição dicionarizada.

12. Ramos e Silva (2018) e Reis (2018).

13. Produto da tradução do conhecimento, que busca superar a barreira da linguagem científica, aproximando produção acadêmica e gestão pública. 


\section{DISCUSSÃO DOS RESULTADOS}

O quadro 1 sintetiza os elementos-chave dos estudos.

QUADRO 1

Síntese dos resultados

\begin{tabular}{|c|c|c|c|c|}
\hline & Estudo & Área & Metodologia & Principais elementos \\
\hline \multirow{6}{*}{ Artigos } & Dias et al. (2015) & Saúde & Revisão de literatura & $\begin{array}{l}\text { Apresenta quatro estratégias para potencializar o uso de evidências } \\
\text { no sistema de saúde. }\end{array}$ \\
\hline & $\begin{array}{l}\text { Campos, } \\
\text { Onocko-Campos e } \\
\text { Barrio (2013) }\end{array}$ & Saúde mental & Discussão teórica & $\begin{array}{l}\text { Reflete sobre categorias de evidências e evidências qualitativas, } \\
\text { contextualizando a prática da saúde mental. } \\
\text { Problematiza a produção de evidências em saúde, quando } \\
\text { financiadas por grandes empresas, e defende a participação de } \\
\text { atores sociais para validar evidências quando houver valores } \\
\text { em conflito. }\end{array}$ \\
\hline & $\begin{array}{l}\text { Pedroso, Juhásová e } \\
\text { Hamann (2019) }\end{array}$ & $\begin{array}{l}\text { Uso de álcool } \\
\text { e drogas }\end{array}$ & Revisão de literatura & $\begin{array}{l}\text { Analisa desafios de adaptação de práticas desenvolvidas com bases } \\
\text { em evidências. Argumenta que essas ações devem considerar as } \\
\text { vulnerabilidades sociais do Brasil, distintas dos países de origem das } \\
\text { evidências examinadas. }\end{array}$ \\
\hline & $\begin{array}{l}\text { Picon, Beltrame e } \\
\text { Banta (2013) }\end{array}$ & Saúde pública & Análise descritiva & $\begin{array}{l}\text { Descreve o desenvolvimento de diretrizes nacionais para tratamento } \\
\text { de doenças, elaboradas com base em evidências, validadas pela } \\
\text { comunidade epistêmica. }\end{array}$ \\
\hline & Campos (2009) & Educação & Revisão de literatura & $\begin{array}{l}\text { Problematiza o uso de evidências em educação, destacando que } \\
\text { algumas questões fundamentais são mais bem equalizadas por } \\
\text { meio dos processos sociais. }\end{array}$ \\
\hline & $\begin{array}{l}\text { Carneiro e } \\
\text { Sandroni (2017) }\end{array}$ & Política pública & Pesquisa qualitativa & $\begin{array}{l}\text { Reflete sobre as PPBEs, analisando a articulação entre ciência } \\
\text { e políticas públicas a partir da percepção de gestores federais. } \\
\text { Discute os limites e possibilidades da contribuição do conhecimento } \\
\text { científico às políticas públicas. }\end{array}$ \\
\hline \multirow{4}{*}{ Dissertações } & Soares (2019) & Segurança pública & Pesquisa qualitativa & $\begin{array}{l}\text { Avalia a implementação de programas baseados em evidências } \\
\text { e conclui que o desenho baseado em evidências contribui } \\
\text { para a efetividade. }\end{array}$ \\
\hline & Gemael (2016) & Ecologia & Pesquisa qualitativa & $\begin{array}{l}\text { Discute a apropriação de gestores e políticos de evidências } \\
\text { científicas; aponta determinantes para o distanciamento } \\
\text { identificado entre ambos, e propõe alternativas de aproximação. }\end{array}$ \\
\hline & Maranhão (2017) & Educação & Pesquisa documental & $\begin{array}{l}\text { Toma como modelos programas elaborados com base em } \\
\text { evidências, para análise de uma intervenção para a qual propõe } \\
\text { alternativas de melhoria, aproximando-a dos modelos. }\end{array}$ \\
\hline & Ramos (2017) & Saúde pública & Pesquisa documental & $\begin{array}{l}\text { Reúne evidências disponíveis em revisões sistemáticas para } \\
\text { elaborar modelo de análise da implementação de estratégia de } \\
\text { saúde pública. }\end{array}$ \\
\hline
\end{tabular}

Elaboração das autoras.

Um dos artigos (Campos, Onocko-Campos e Barrio, 2013) discute a produção das evidências, sua hierarquização e o risco de reificação de evidências quantitativas, trazendo dificuldades para campos em que esses estudos não são abundantes ou possíveis.

Cinco estudos se dedicam a analisar estratégias de uso/não uso das evidências. Três o fazem a partir de sua problematização, conforme descrito a seguir.

- Pedroso, Juhásová e Hamann (2019) argumentam sobre a dificuldade para incluir evidências científicas como um elemento relevante na formulação de intervençôes, ao observarem que as decisôes são mais orientadas pelos apelos morais e normas culturais do que por evidências no campo da "guerra às drogas". 
- Campos (2009) analisa o uso de evidências diante de um quadro mais amplo, que questiona os objetivos da pesquisa em educaçáo e a utilidade dos resultados obtidos face às questóes estruturais que devem ser enfrentadas nesse campo.

- Carneiro e Danton (2012) identificaram, entre gestores, a coexistência de duas visôes sobre ciência: como narrativa apartada da política e como campo legítimo da geração de dados e tecnologias importantes para fundamentação das disputas inerentes à prática governamental. Argumentam que a percepção dos gestores sobre o lugar da evidência científica interfere no sucesso dessa abordagem, e concluem que a contribuição das PPBEs se dá mais na criação de legitimidade ou no fortalecimento de temas na agenda do que na seleção das melhores opçóes de política.

Os dois últimos criticam a adoção irrefletida da abordagem das PPBEs e os pressupostos de que a evidência científica agregaria soluçôes inéditas para a gestão pública. Outros dois estudos abordam o tema a partir da discussão sobre quais seriam as melhores estratégias para estimular o uso de evidências na tomada de decisão (Dias et al., 2015), sintetizadas nas seguintes possibilidades: i) produzir e disseminar sínteses de evidência com linguagem adaptada aos diferentes públicos; ii) estimular o uso do jornalismo e de outras formas de comunicação para ampliar e disseminar conhecimento científico; iii) utilizar plataforma virtual para disseminar conhecimento científico; e iv) promover interação entre pesquisadores e tomadores de decisão. Essas possibilidades são também apontadas nas conclusóes de Gemael (2016) para a incorporação das evidências no campo da gestão de políticas ambientais, ao identificar, no seu estudo, o distanciamento entre esses campos e defender a aproximação entre ambos.

Há, ainda, o debate sobre o desenho de intervençóes baseadas em evidências. $\mathrm{O}$ estudo de Picon, Beltrame e Banta (2013) descreve uma estratégia desenvolvida pelo Ministério da Saúde para enfrentar a judicialização da saúde, a partir da criaçáo de diretrizes nacionais baseadas em evidências científicas, discutidas com a comunidade epistêmica (Costa, 2014) e, posteriormente, distribuídas para a rede pública. Maranhão (2017) adota programas baseados em evidências no campo da educação como referência para análise de uma iniciativa e proposição de açóes que a tornem mais efetiva, com a incorporação de elementos identificados nos modelos.

Por fim, dois estudos analisam/avaliam intervenções. Ramos (2017) usa um modelo elaborado a partir de evidências científicas para análise da implementação da estratégia de regionalização do atendimento de saúde nos estados que adotaram o modelo do governo federal; e Soares (2019) avalia a implementação das PPBEs e conclui que o fato de serem baseadas em evidências contribui para a efetividade de seus resultados.

\section{CONSIDERAÇÕES FINAIS}

A análise da produção acadêmica evidenciou que a produção sobre as PPBEs ainda se mostra numericamente pouco expressiva no Brasil. $\mathrm{O}$ interesse pelo tema parece ainda náo ter se refletido na produção acadêmica brasileira, e chama a atenção a ausência de análises sobre as iniciativas governamentais dos últimos anos. A tônica mais presente parece ser a tentativa de criação de pontes para o diálogo com a gestão pública. A estratégia de aproximação se daria por meio da "tradução" da linguagem acadêmica e da disseminação de sua produção para alcançar gestores públicos, revelando que a superação do principal desafio estaria na remoção das barreiras de acesso às evidências científicas, transformando a linguagem e ampliando a disponibilidade. Identificamos também uma discussão crítica sobre o uso de evidências nas políticas públicas. Aqui, a incorporação das evidências precisa 
superar outros desafios, como o reconhecimento dos limites de seu alcance face a problemas estruturais e complexos enfrentados pelas políticas públicas.

\section{REFERÊNCIAS}

CAMPOS, G. W. de S.; ONOCKO-CAMPOS, R. T.; BARRIO, L. R. del. Políticas e práticas em saúde mental: as evidências em questão. Ciência e Saúde Coletiva, v. 18, n. 10, p. 2797-2805, out. 2013.

CAMPOS, M. Para que serve a pesquisa em educação? Cadernos de Pesquisa, São Paulo, v. 39, n. 136, p. 269-283, abr. 2009.

CARNEIRO, M. J. T.; DANTON, T. Agricultura e biodiversidade nas ciências sociais brasileiras: alimentando a comunicação entre ciência e políticas públicas. Sociologias, v. 14, n. 30, p. 252-289, maio 2012.

CARNEIRO, M. J. T.; SANDRONI, L. T. Science and policy on the perspective of public managers: relativizing borders. Sociedade e Estado, v. 33, n. 1, p. 41-61, 1ํ jan. 2018.

COSTA, N. do R. Comunidade epistêmica e a formação da reforma sanitária no Brasil. Physis: Revista de Saúde Coletiva, v. 24, n. 3, p. 809-829, 2014.

DAVIES, H. T. O.; NUTLEY, S. M. The rise and rise of evidence in health care. Public Money and Management, v. 19, n. 1, p. 9-16, jan. 1999.

DAVIES, P. Is evidence-based government possible? London: Jerry Lee Lecture, 2004.

DIAS, R. I. da S. C. et al. Estratégias para estimular o uso de evidências científicas na tomada de decisão. Cadernos de Saúde Coletiva, v. 23, n. 3, p. 316-322, set. 2015.

GEMAEL, M. Evidências científicas como base das políticas públicas para a conservaçáo da biodiversidade no Brasil. Cuiabá: Editora da UFMT, 2016.

HEAD, B. W. Three lenses of evidence-based policy. Australian Journal of Public Administration, v. 67 , n. 1 , p. 1-11, mar. 2008.

MARANHÃO, M. O pacto pela educação em Recife: desafios na implementação da gestão pedagógica com base em evidências. 2017. Dissertação (Mestrado) - Universidade Federal de Juiz de Fora, Juiz de Fora, 2017.

OLIVER, K. et al. A systematic review of barriers to and facilitators of the use of evidence by policymakers. BMC Health Services Research, v. 14, n. 2, 3 jan. 2014.

PEDROSO, R. T.; JUHÁSOVÁ, M. B.; HAMANN, E. M. Evidence-based science in public policies for reinventing alcohol and drugs use prevention. Interface: Communication, Health, Education, v. 23, 2019.

PICON, P. D.; BELTRAME, A.; BANTA, D. National guidelines for high-cost drugs in Brazil: achievements and constraints of an innovative national evidence-based public health policy. International Journal of Technology Assessment in Health Care, v. 29, n. 2, p. 198-206, 2013. 
RAMOS, M. O processo de regionalizaçáo via Coap informado por evidências: estamos no caminho certo? 2017. Dissertação (Mestrado) - Universidade de Brasília, Brasília, 2017.

RAMOS, M. C.; SILVA, E. N. da. Como usar a abordagem da política informada por evidência na saúde pública? Saúde em Debate, v. 42, n. 116, p. 296-306, jan. 2018.

REIS, G. R. A utilização do uso de evidências científicas como política pública para a otimização na disponibilidade de vagas em UTI no Tocantins. 2018. Dissertação (Mestrado) - Universidade Federal do Tocantins, Palmas, 2018.

SOARES, T. Políticas públicas e prevençáo da violência no desenvolvimento infantil baseada em evidências: uma análise da implementação dos programas ACT e Conte Comigo na cidade de Pelotas, RS. 2019. Dissertação (Mestrado) - Universidade Federal do Rio Grande do Sul, Porto Alegre, 2019. 
